\title{
Plant nematodes in South Africa. 7. A check list of plant nematodes from the Fynbos Biome, with a description of Helicotylenchus curatus
}

sp. $n$.

\author{
M. Marais, E. van den Berg, A. Swart and L.R. Tiedt
}

\begin{abstract}
Marais, M., E. van den Berg, A. Swart and L.R. Tiedt. 2004. Plant nematodes in South Africa. 7. A check list of plant nematodes from the Fynbos Biome, with a description of Helicotylenchus curatus sp. n. Koedoe 47(1): 67-78. Pretoria. ISSN 0075-6458.
\end{abstract}

\begin{abstract}
Plant nematodes recorded during surveys in the Fynbos Biome are listed and a new Helicotylenchus species is described. Helicotylenchus curatus $\mathrm{sp}$. $\mathrm{n}$. is characterised by stylet length (42-46 $\mu \mathrm{m}$ in females, $37-40 \mu \mathrm{m}$ in males), presence of two rudimentary subdorsal and two rudimentary subventral lobes on the labial disc, first lip annulus divided into six sectors, presence of fasciculi and presence of males. Nine families represented by 32 genera and 152 species were identified from the Fynbos Biome. The genera Criconema, Helicotylenchus, Hemicycliophora, Rotylenchus, Scutellonema and Xiphinema were found in more than $30 \%$ of the localities, whereas Caloosia, Criconemoides, Ditylenchus, Geocenamus, Hemicriconemoides, Heterodera, Hoplolaimus, Longidorus, Meloidogyne, Mesocriconema, Ogma, Paralongidorus, Paratrichodorus, Paratylenchus, Pratylenchoides, Pratylenchus, Rotylenchulus, Trichodorus and Tylenchorhynchus were found at fewer localities. The genera Anguina, Hirschmaniella, Histotylenchus and Zygotylenchus were each identified from a single locality.
\end{abstract}

Key words: biodiversity, Fynbos, Helicotylenchus, plant nematodes, new species, South Africa, taxonomy.

M. Marais, E. van den Berg, A. Swart, Nematology Unit, Biosystematics Division, ARCPlant Protection Research Institute, Private Bag X134, Queenswood, 0121 Republic of South Africa; L.R. Tiedt, Laboratory for Electron Microscopy, North West University, Potchefstroom Campus, Potchefstroom, 2520 Republic of South Africa.

\section{Introduction}

In 1987, the South African Plant-Parasitic Nematode Survey (SAPPNS) programme was initiated with the aim to make a comprehensive assessment of the nematode biodiversity resources of South Africa. One of the objectives of the SAPPNS is to compile an inventory of the plant-parasitic nematodes of South Africa. Although nematodes constitute an abundant and highly diverse group of invertebrates, very little is known about their diversity in fynbos. The checklist reflects on collecting done by the Agricultural Research Council, Western Cape Nature Conservation Board, Rand Afrikaans University and University of Stellenbosch (Heyns 1971; Kleynhans et al. 1996; Marais \& Swart 1999; Van den Berg et al. 2003; Van den Berg \& Tiedt
2000) during the past 30 years. The Fynbos Biome is one of the biomes defined by Low \& Rebelo (1996). In South Africa, vegetation structure and climate mostly define these ecological zones. The Fynbos Biome is considered by many to be synonymous with the Cape Floral Kingdom, but this biome refers only to the two vegetation groups, viz., Fynbos and Renosterveld within the region (Low \& Rebelo 1996). Renosterveld is characterised by the dominance of members of the Asteraceae, specifically one species Elytropappus rhinocerotis (renosterbos), from which the vegetation type gets its name. Although renosterbos is the characteristic dominant plant, other plants are also prominent. These plants are all shrubs, characterised by small, tough grey leaves (Rebelo 1996a). The various Fynbos vegetation types 
comprise most of the area of the Fynbos Biome. Fynbos is characterised by the presence of three elements, viz., a component belonging to the Cape Reed family (Restionaceae), an ericoid or heath component and a proteoid component. Fynbos is also characterised by the presence of a number of endemic or near-endemic plant families, viz., Bruniaceae (Blacktips), Geissolomaceae (Guyalone), Grubbiaceae (Sillyberry), Penaeaceae (Brickleaf), Retziaceae (Buttbush), Roridulaceae (Dewstick) and Stilbaceae (Candlestick) (Rebelo 1996b).

Helicotylenchus is a cosmopolitan genus with more than 200 species described (Marais 2001). This genus is found in all the biomes of South Africa. Thirty-one species of Helicotylenchus have been reported from South Africa. This paper is the result of an ongoing investigation on the genus Helicotylenchus (Marais 1993, 1998, 2001; Marais \& Buckley 1992; Marais \& Quénéhervé 1996; Marais \& Quénéhervé 1999; Marais et al. 2000; Van den Berg \& Marais 1995; Van den Berg et al. 2003).

\section{Material and methods}

Soil samples were collected with a garden trowel, soil auger or spade to a depth of about $20-25 \mathrm{~cm}$ at each locality. Samples were collected in unprotected areas such as farms and plantations but also in nature reserves, wilderness areas and national parks. Nematodes were extracted from $250 \mathrm{~cm}^{3}$ of soil, killed in water, preserved in FAA, TAF or FPG and mounted in anhydrous glycerine (Hooper \& Evans 1993; Jenkins 1964; Kleynhans 1997; Koen \& Furstenberg 1970; Netscher \& Seinhorst 1969; Seinhorst 1959).

To obtain females of Meloidogyne for identification, subsamples of each soil sample, in which root-knot nematode juveniles were found, were planted to tomato seedlings (cv. UC82B or Roma VF) in a greenhouse. After six weeks, females were extracted from the roots and mounted in anhydrous glycerine (Kleynhans 1991). For scanning electron microscopy, FPG-preserved specimens were used after dehydration in increasing concentrations of acetone. Following conventional critical-point drying and gold/palladium-coating $(15 \mathrm{~nm})$, specimens were viewed with a Quanta 200 ESEM.

\section{Results and discussion}

The classification of nematodes followed is based on Maggenti et al. (1988) for Tylenchina, Hunt (1993) for Aphelenchina and Longidoridae and Decraemer (1995) for Trichodoriae. Nine families represented by 32 genera and 152 species were identified from the Fynbos biome (Table 1). The genera Criconema, Helicotylenchus, Scutellonema, Rotylenchus and Xiphinema were the most common and were found at $40 \%$, $35 \%, 31 \%, 30 \%$ and $30 \%$ of the localities, respectively. The predominance of records in the Mountain Fynbos vegetation type is a consequence of the number of samples collected in this vegetation type (Table 1). Mountain Fynbos is also the vegetation type with the highest total area $\left(27461 \mathrm{~km}^{2}\right)$ of the ten Fynbos vegetation types (Low \& Rebelo 1996).

\section{Taxonomy}

\section{Helicotylenchus curatus sp. $\boldsymbol{n}$.}

Figs. 1 \& 2

\section{Measurements}

Holotype female: $\mathrm{L}=933 \mu \mathrm{m} ; \mathrm{a}=26.4$; $\mathrm{c}=$ $43.8 ; \mathrm{c}^{\prime}=1.2$; stylet length $=42 \mu \mathrm{m} ; \mathrm{m}=$ $53 \%$; V $=62 \%$.

Paratype females $(n=9): \mathrm{L}=1040 \pm 110.1$ (839-1262) $\mu \mathrm{m} ; \mathrm{a}=23.0 \pm 2.9$ (17.5-27.5); $\mathrm{c}=44.1 \pm 4.5(38.1-52.0) ; \mathrm{c}^{\prime}=1.0 \pm 0.2$ $(0.8-1.2)$; stylet length $=44 \pm 1.7(42-46)$; $\mathrm{m}=54 \pm 1.4(52-56) \% ; \mathrm{V}=61 \pm 1.8$ (58-64) \%.

Paratype males $(n=5): \mathrm{L}=864 \pm 69.6$ (760-944) $\mu \mathrm{m} ; \mathrm{a}=36.2 \pm 3.4$ (32.5-39.2); $=30.0 \pm 2.9(27.0-34.5) ; \mathrm{c}^{\prime}=1.8 \pm 0.4$ (1.42.6); stylet length $=39 \pm 1.4 \quad(37-40) \mu \mathrm{m}$; $\mathrm{m}=57 \pm 3.5(52-60) \%$; spicule length $=33$ \pm 0.9 (32-34) $\mu \mathrm{m}$; gubernaculum length $=10$ \pm 0.7 (9-11) $\mu \mathrm{m}$.

Female: Habitus spiral. Lip region $7 \pm 0.5$ (6-8) $\mu \mathrm{m}$ high and $10 \pm 0.9(10-13) \mu \mathrm{m}$ wide, truncate with distinct labial disc, six to seven annuli, basal annuli bulging out. 
Table 1

Plant nematodes found associated with the Fynbos Biome

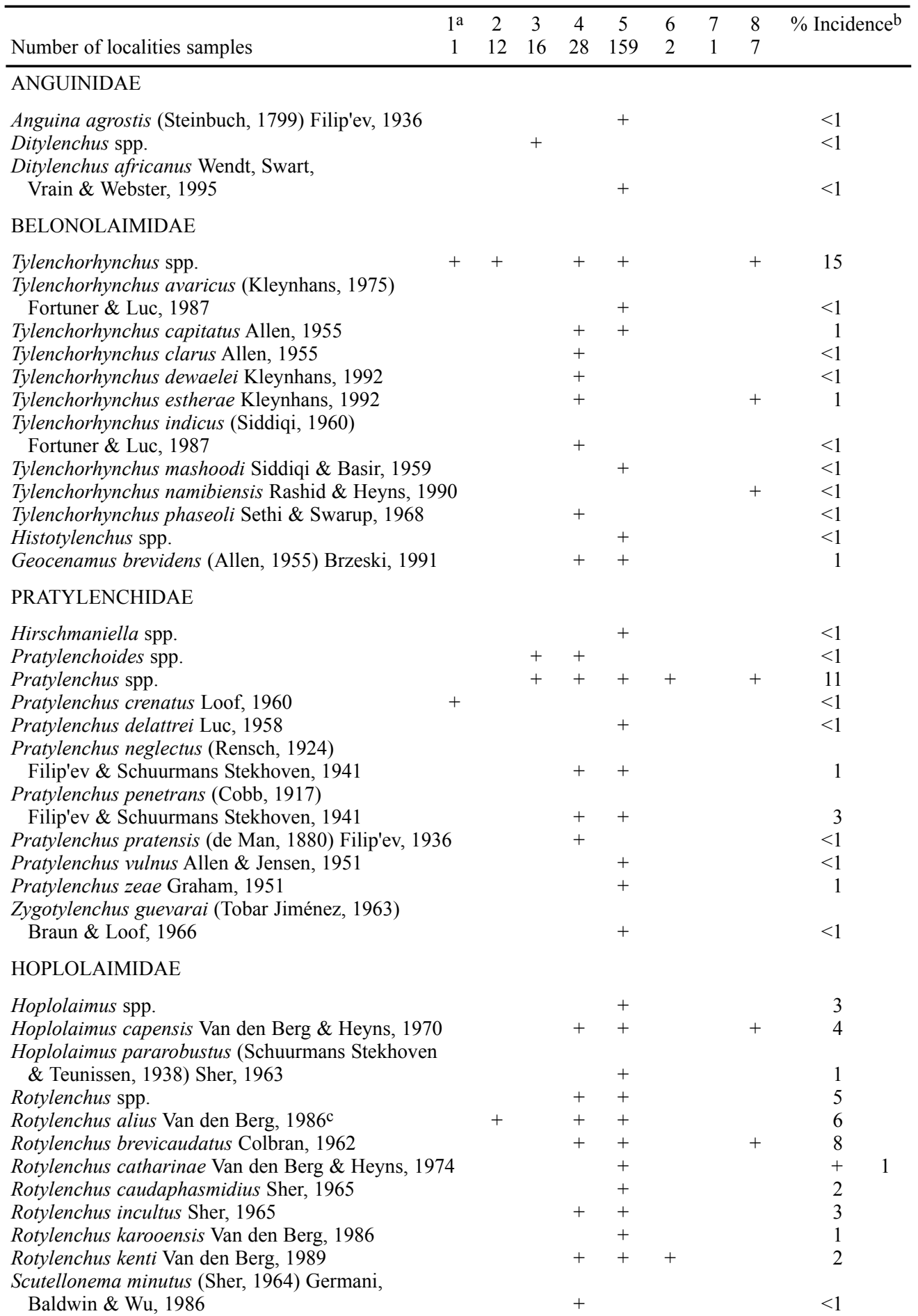


Table 1 (continued)

\begin{tabular}{|c|c|c|c|c|c|c|c|c|}
\hline Number of localities samples & $\begin{array}{c}2 \\
12 \\
\end{array}$ & $\begin{array}{c}3 \\
16 \\
\end{array}$ & $\begin{array}{c}4 \\
28 \\
\end{array}$ & $\begin{array}{c}5 \\
159 \\
\end{array}$ & $\begin{array}{l}6 \\
2\end{array}$ & $\begin{array}{l}7 \\
1 \\
\end{array}$ & $\begin{array}{l}8 \\
7\end{array}$ & $\%$ Incidence $^{b}$ \\
\hline Rotylenchus unisexus Sher, 1965 & + & & + & + & & & & 6 \\
\hline Rotylenchus usitatus Van den Berg \& Heyns, 1974 & & & & + & & & & $<1$ \\
\hline Helicotylenchus spp. & + & & + & + & + & & + & 22 \\
\hline Helicotylenchus californicus Sher, 1966 & & & + & + & & & & 2 \\
\hline Helicotylenchus cavenessi Sher, 1966 & & & + & & & & & $<1$ \\
\hline Helicotylenchus curatus sp. $\mathrm{n}$. & & & & + & & & & $<1$ \\
\hline \multicolumn{9}{|l|}{ Helicotylenchus digonicus Perry in Perry, } \\
\hline Darling \& Thorne, 1959 & & & + & + & & & & 2 \\
\hline Helicotylenchus dihystera (Cobb, 1893) Sher, 1961 & & & + & + & + & & & 4 \\
\hline Helicotylenchus exallus Sher, 1966 & & & & + & & & + & 6 \\
\hline Helicotylenchus hydrophilus Sher, 1966 & & & & + & & & & $<1$ \\
\hline Helicotylenchus minzi Sher, 1966 & & & & + & & & & 4 \\
\hline Helicotylenchus paraplatyurus Siddiqi, 1972 & & & + & + & & & & 1 \\
\hline \multicolumn{9}{|l|}{ Helicotylenchus pseudorobustus (Steiner, 1914) } \\
\hline Golden, 1956 & & & + & & & & & 1 \\
\hline Helicotylenchus serenus Siddiqi, 1963 & & & + & + & & & & 1 \\
\hline Scutellonema spp. & + & & + & + & & & + & 8 \\
\hline Scutellonema bizanae Van den Berg \& Heyns, 1973 & + & & + & + & & & + & 13 \\
\hline \multicolumn{9}{|l|}{ Scutellonema brachyurus (Steiner, 1938) } \\
\hline Andrássy, 1958 & & & + & + & & & + & 9 \\
\hline Scutellonema nigermontanum Van den Berg, 1990 & & & & + & & & & $<1$ \\
\hline Scutellonema tsitsikamense Van den Berg, 1976 & & & & + & & & & 1 \\
\hline Scutellonema unum Sher, 1964 & & & + & & & & & $<1$ \\
\hline Rotylenchulus spp. & + & & & & & & & 1 \\
\hline Rotylenchulus borealis Loof \& Oostenbrink, 1962 & & & & + & & & & 2 \\
\hline $\begin{array}{l}\text { Rotylenchulus macrodoratus Dasgupta, } \\
\text { Raski \& Sher, } 1968\end{array}$ & & & & + & & & & 1 \\
\hline Rotylenchulus parvus (Williams, 1960) Sher, $1961+$ & & + & + & + & & & & 5 \\
\hline Rotylenchulus reniformis Linford \& Oliveira, 1940 & & & & + & & & & \\
\hline \multicolumn{9}{|l|}{ HETERODERIDAE } \\
\hline Heterodera spp. & & & & + & & & & 1 \\
\hline Heterodera trifolii Goffart, 1932 & & & & + & & & & $<1$ \\
\hline Meloidogyne spp. & & + & + & + & & & & 5 \\
\hline Meloidogyne hapla Chitwood, 1949 & & & & + & & & + & 3 \\
\hline \multicolumn{9}{|l|}{ Meloidogyne incognita (Kofoid \& White, 1919) } \\
\hline Chitwood, 1949 & & & & + & & & & 3 \\
\hline Meloidogyne javanica (Treub, 1885) Chitwood, 1949 & & & + & + & & & + & 3 \\
\hline \multicolumn{9}{|l|}{ CRICONEMATIDAE } \\
\hline Criconema spp. & + & & + & + & & & + & 13 \\
\hline Criconema ananas (Heyns, 1970) Siddiqi, 1986 & & & + & & & & & $<1$ \\
\hline \multicolumn{9}{|l|}{ Criconema corbetti (De Grisse, 1967) } \\
\hline Raski \& Luc, 1985 & & & & + & & & & 3 \\
\hline \multicolumn{9}{|l|}{ Criconema crassianulatum (de Guiran, 1963) } \\
\hline Raski \& Luc, 1985) & & & & + & & & & 1 \\
\hline \multicolumn{9}{|l|}{ Criconema duplicivestitum (Andrássy, 1963) } \\
\hline Raski \& Luc, 1985 & & & & + & & & & 11 \\
\hline Criconema indigenae Van den Berg \& Meyer, $1991^{\mathrm{c}}$ & & & & + & & & & 1 \\
\hline \multicolumn{9}{|l|}{ Criconema mutabile (Taylor, 1936) } \\
\hline Raski \& Luc, 1987 & + & & + & + & + & & + & 4 \\
\hline Criconema proteae Van den Berg \& Meyer, 1991c & & & & + & & & & 1 \\
\hline \multicolumn{9}{|l|}{ Criconema sanctifrancisci (Van den Berg \& } \\
\hline Heyns, 1977) Raski \& Luc, 1985 & & & & + & & & + & 11 \\
\hline Criconema simplex Marais \& Van den Berg, 1996c & & & & + & & & & 1 \\
\hline
\end{tabular}


Table 1 (continued)

\begin{tabular}{|c|c|c|c|c|c|c|c|c|c|}
\hline Number of localities samples & $\begin{array}{c}1^{\mathrm{a}} \\
1\end{array}$ & $\begin{array}{c}2 \\
12 \\
\end{array}$ & $\begin{array}{c}3 \\
16 \\
\end{array}$ & $\begin{array}{c}4 \\
28 \\
\end{array}$ & $\begin{array}{c}5 \\
159 \\
\end{array}$ & $\begin{array}{l}6 \\
2\end{array}$ & $\begin{array}{l}7 \\
1\end{array}$ & $\begin{array}{l}8 \\
7\end{array}$ & $\%$ Incidence $^{b}$ \\
\hline Criconema sirgeli Van den Berg \& Meyer, $1987^{c}$ & & & & & + & & & & $<1$ \\
\hline Criconemoides parvus Raski, 1952 & & & & + & + & & & & 2 \\
\hline Hemicriconemoides spp. & & & & & + & & & & $<1$ \\
\hline Hemicriconemoides brachyurus (Loos, 1949) & & & & & & & & & \\
\hline Chitwood \& Birchfield, 1957 & & + & & + & + & & & + & 6 \\
\hline Hemicriconemoides capensis Van den Berg, 1990 & & & & & + & & & & $<1$ \\
\hline $\begin{array}{l}\text { Hemicriconemoides cedrusmontanus } \\
\text { Van den Berg \& Meyer, 1991 }\end{array}$ & & & & & + & & & & $<1$ \\
\hline $\begin{array}{l}\text { Mesocriconema spp. } \\
\text { Mesocriconema ferniae (Luc, 1959) }\end{array}$ & & + & + & + & + & & + & + & 12 \\
\hline $\begin{array}{l}\text { Loof \& De Grisse, } 1989 \\
\text { Mesocriconema obtusicaudatum (Heyns, 1962) }\end{array}$ & & & & & + & & & & 3 \\
\hline Loof \& De Grisse, 1989 & + & & & + & + & & & & 3 \\
\hline Mesocriconema sphaerocephalum (Taylor, 1936) & & & & & & & & & \\
\hline Loof \& De Grisse, 1989 & & & & & + & & & & 1 \\
\hline Mesocriconema thabaum Van den Berg, $1996^{c}$ & & & & & + & & & & $<1$ \\
\hline Mesocriconema xenoplax (Raski, 1952) & & & & & & & & & \\
\hline Loof \& De Grisse, 1989 & & + & & & + & & & + & 1 \\
\hline Ogma spp. & & + & & & & & & & $<1$ \\
\hline Ogma civellae civellae Reay \& Davies, 1998 & & & & + & + & & & & \\
\hline Ogma decalineatum (Chitwood, 1957) & & & & & & & & & \\
\hline Andrássy, 1979 & & & & & + & & & & 1 \\
\hline Ogma inornatum (Van den Berg, 1983) & & & & & & & & & \\
\hline Siddiqi, $1986^{c}$ & & & & + & & & & & $<1$ \\
\hline Ogma rhombosquamatum (Mehta \& Raski, 1971) & & & & & & & & & \\
\hline Andrássy, 1979 & & & & + & & & & + & 1 \\
\hline Ogma squamiferum (Heyns, 1970) Andrássy, 1979c & & & + & + & & & & & 1 \\
\hline $\begin{array}{l}\text { Caloosia exigua Van den Berg, Marais } \\
\& \text { Tiedt, } 2003^{\mathrm{c}}\end{array}$ & & & & & + & & & & \\
\hline Caloosia peculiaris Van den Berg \& Meyer, 1991c & & & & & + & & & & $<1$ \\
\hline Hemicycliophora spp. & & & & + & + & & & + & 9 \\
\hline Hemicycliophora demani Edward \& Rai, 1971 & & & & & + & & & & 2 \\
\hline Hemicycliophora epicharoides Loof, 1968 & & & & & + & & & & $<1$ \\
\hline Hemicycliophora halophila Yeates, 1967 & & & & + & + & & & & 1 \\
\hline Hemicycliophora labiata Colbran, 1960 & & & & + & + & & + & & 7 \\
\hline Hemicycliophora natalensis Loof \& Heyns, 1969 & & + & & & + & & & & 1 \\
\hline Hemicycliophora nullinca Van den Berg, 1987 & & + & & & & & & & $<1$ \\
\hline Hemicycliophora peca Van den Berg, 1987c & & & & & + & & & & $<1$ \\
\hline Hemicycliophora stiaani Van den Berg \& & & & & & & & & & \\
\hline Tiedt, $1999^{c}$ & & & & & + & & & & $<1$ \\
\hline Hemicycliophora typica de Man, 1921 & & & & & + & & & & 1 \\
\hline Hemicycliophora wesca Van den Berg \& & & & & & & & & & \\
\hline Meyer, $1987^{\mathrm{c}}$ & & & & & + & & & & $<1$ \\
\hline TYLENCHULIDAE & & & & & & & & & \\
\hline Paratylenchus spp. & & + & & & + & & & & $<1$ \\
\hline Paratylenchus arculatus Luc \& de Guiran, 1962 & & & & & + & & & & $<1$ \\
\hline Paratylenchus elachistus Steiner, 1949 & & & & & + & & & & $<1$ \\
\hline Paratylenchus projectus Jenkins, 1956 & & + & & & + & & & & 1 \\
\hline Paratylenchus vandenbrandei De Grisse, 1962 & & & & & + & & & & $<1$ \\
\hline TRICHODORIDAE & & & & & & & & & \\
\hline Trichodorus spp. & & & & & + & & & & 5 \\
\hline Trichodorus iuventus Decraemer \& Marais, 2000c & & & & & + & & & & $<1$ \\
\hline
\end{tabular}


Table 1 (continued)

\begin{tabular}{lccccccccc}
\hline & $1 \mathrm{a}$ & 2 & 3 & 4 & 5 & 6 & 7 & 8 & \% Incidence $^{\mathrm{b}}$ \\
Number of localities samples & 1 & 12 & 16 & 28 & 159 & 2 & 1 & 7 & \\
\hline
\end{tabular}

Trichodorus philipi De Waele \&

Van Mieghem, 1990c

Trichodorus vandenbergae De Waele \& Kilian, 1992

Paratrichodorus spp.

Paratrichodorus lobatus (Colbran. 1956)

Siddiqi, 1974

Paratrichodorus minor (Colbran. 1956)

Siddiqi, 1974

Paratrichodorus porosus (Allen, 1957) Siddiqi, 1974

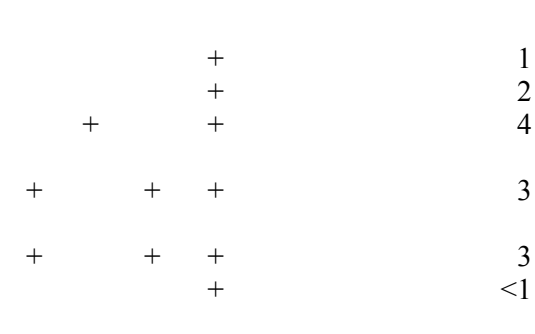

LONGIDORIDAE

Paralongidorus spp.

Paralongidorus capensis (Heyns, 1967)

Liebenberg, Heyns \& Swart, 1993

Paralongidorus christiani Liebenberg, Heyns

\& Swart, 1993 ${ }^{\mathrm{c}}$

Paralongidorus costatus (Jacobs \& Heyns, 1987)

Siddiqi, Baujard \& Mounport, 1993

Paralongidorus deborae (Jacobs \& Heyns, 1982)

Luc \& Doucet, 1984

Paralongidorus spasskii Heyns, 1972

Longidorus spp.

Longidorus fursti Heyns, Coomans, Hutsebaut \& Swart, 1987

Longidorus jagerae Heyns \& Swart, 1998

Longidorus juvenilis Dalmasso, 1969

Longidorus pisi Edward, Misra \& Singh, 1964

Xiphinema spp.

Xiphinema americanum Cobb, 1913

Xiphinema barbercheckae Coomans \& Heyns, 1985

Xiphinema bolandium Coomans \& Heyns, 1985

Xiphinema capense Coomans \& Heyns, 1985

Xiphinema diffusum Lamberti \&

Bleve-Zacheo, 1979

Xiphinema elongatum Schuurmans Stekhoven \&

Teunissen, 1938

Xiphinema hardingi Joubert, Kruger \& Heyns, 1988

Xiphinema krugi Lordello, 1955

Xiphinema mampara Heyns, 1979

Xiphinema meridianum Heyns, 1971

Xiphinema mluci Heyns, 1976

Xiphinema pachtaicum (Tulaganov, 1938)

Kir'yanova, 1951

Xiphinema parvistilus Heyns, 1971

Xiphinema ripogranum Hutsebaut, Heyns \&

Coomans, $1988^{\mathrm{c}}$

Xiphinema vanderlindei Heyns, 1962

Xiphinema variabile Heyns, 1966

Xiphinema vitis Heyns, 1974

Xiphinema zulu Heyns, 1965

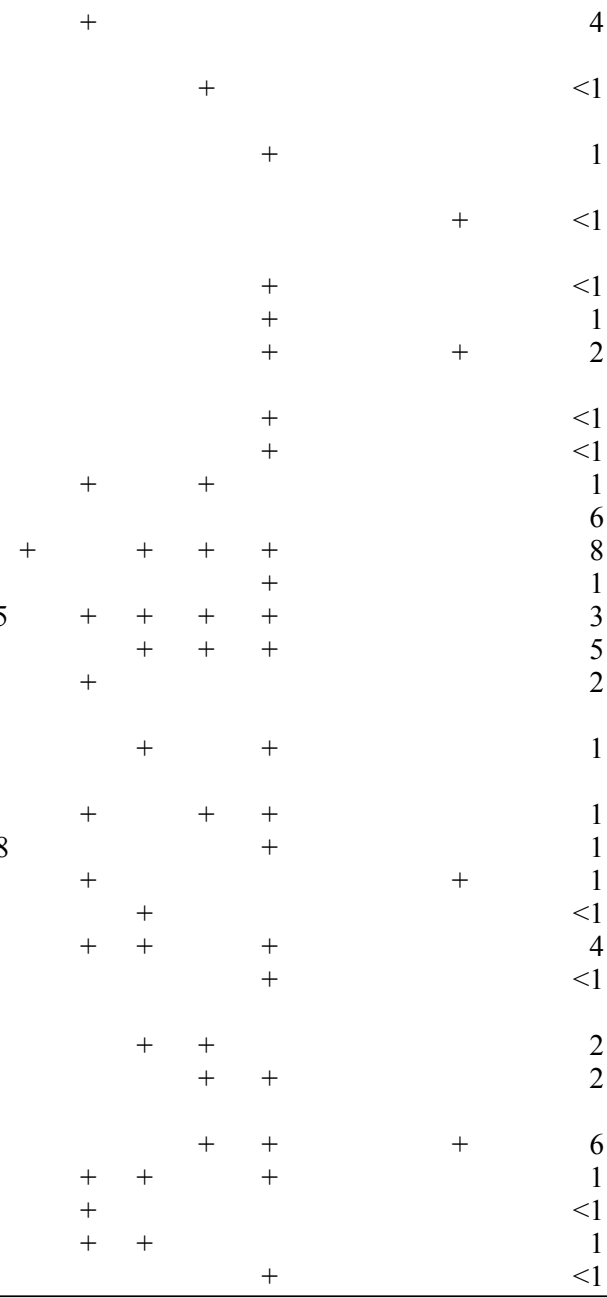

4

$<1$

1

$<1$

$<1$

2

$<1$

$<1$

6

8

5

2

1

1

1

1

1

4
$<1$

2

2

6

1

$<1$

$<1$

a Vegetation types of the Fynbos Biome according to Low \& Rebelo (1996) $(1$ = Escarpment Mountain Renosterveld, 2 = Central Mountain Renosterveld, 3 = West Coast Renosterveld, $4=$ South and South-west Coast Renosterveld, 5 = Mountain Fynbos, 6 = Grassy Fynbos, 7 = Laterite Fynbos, 9 = Sand Plain Fynbos)

$\mathrm{b}$ Percentage incidence in total number of samples collected during surveys.

c Nematodes described from the Fynbos Biome. 

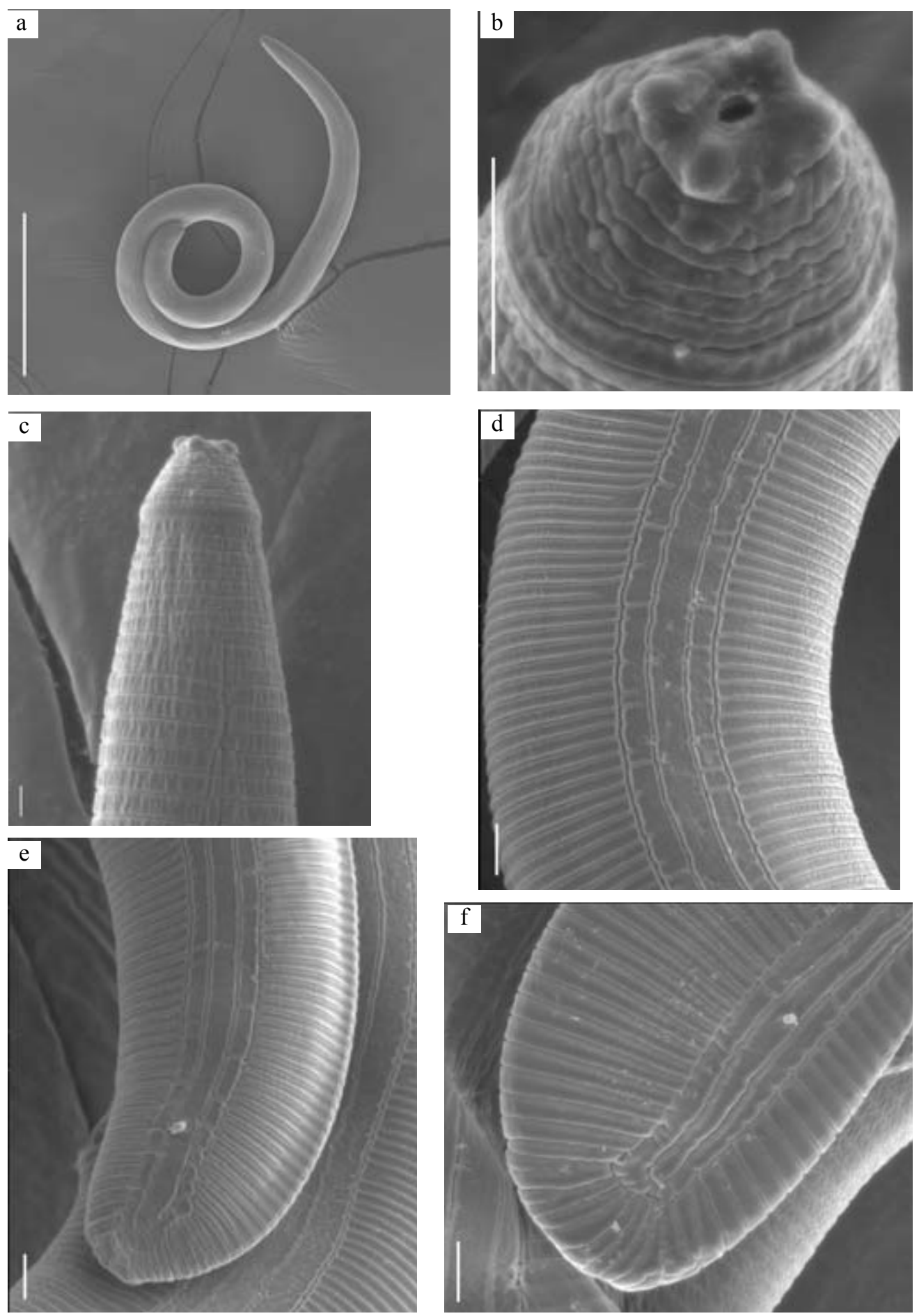

Fig. 1. Helictylenchus curatus sp. n. Female. (a) Habitus. (b) En face view, lip region. (c) Lateral view, anterior part of body. (d) Lateral field. (e-f) Posterior part of body. (Scale bar: $a=100 \mu \mathrm{m}, b-e=4 \mu \mathrm{m}$ ). 
Labial disc rectangular in en face view, with two rudimentary subdorsal and two rudimentary subventral lobes. First lip annulus divided into six sectors, two lateral, two subventral and two subdorsal. Outer margins of the well-developed labial framework extend $4 \pm$ 0.2 (3-4) $\mu \mathrm{m}$ backward from basal plate. Anterior cephalids three to four annuli posterior to basal plate, posterior cephalids 11 to 13 annuli posterior to anterior cephalids. Stylet slender, stylet knobs $4 \pm 0.5(3-4) \mu \mathrm{m}$ high and $7 \pm 1.3(6-10) \mu \mathrm{m}$ wide; anterior faces rounded $(14 \%)$, flattened $(29 \%)$, indented $(14 \%)$ or flattened and slightly inclined backward (43\%). Position of dorsal gland opening (DGO) $5 \pm 1.7$ (3-9) $\mu \mathrm{m}$ behind stylet knobs. Median bulb oval, 16 \pm 1.4 (14-18) $\mu \mathrm{m}$ long and $12 \pm 1.7$ (10-15) $\mu \mathrm{m}$ wide; valve $4 \pm 0.4(3-4) \mu \mathrm{m}$ long and $3 \mu \mathrm{m}$ wide. Length of oesophagus $151 \pm 13.0(134-175) \mu \mathrm{m}$, with length to end of gland $172 \pm 19.0(154-212) \mu \mathrm{m}$. Oesophagus with $22 \pm 4.3(16-28) \mu \mathrm{m}$ long ventral and dorsal overlap. Excretory pore $154 \pm$ $11.0(142-172) \mu \mathrm{m}$ from front, i.e. at $15 \pm$ 1.3 (14-17) \% of body length. Hemizonid two annuli long located one to three annuli anterior to excretory pore. Hemizonion half an annulus long, located 10 annuli posterior to excretory pore $(n=1)$. Fasciculi present. Width of annulus at midbody $2 \mu \mathrm{m}$. Body width at excretory pore $33 \pm 4.7(26-40) \mu \mathrm{m}$, at midbody $46 \pm 8.2(35-59) \mu \mathrm{m}$ and at anus $24 \pm 4.8$ (18-29) $\mu \mathrm{m}$. Two branches of the reproductive system both functional, length of posterior branch $81 \pm 16.6$ (57-94) \% of corresponding anterior branch length; anterior branch $276 \pm 59.8(165-334) \mu \mathrm{m}$ and posterior branch $199 \pm 38.5$ (156-235) $\mu \mathrm{m}$ long. Spermatheca set off, empty or filled with sperm. Epiptygma folded into vagina. Lateral field $10 \pm 1.7(8-13) \mu \mathrm{m}$ wide; outer two lines areolated opposite oesophagus, crenate or incompletely areolated on rest of body; inner two lines end on tail in a u-shaped $(80 \%)$ or open m-shaped pattern $(20 \%)$. Phasmids located from five annuli posterior to opposite anus. Tail $24 \pm 2.3(20-28) \mu \mathrm{m}$ long, asymmetrical, more curved dorsally, with rounded end, with 13 to 18 ventral annuli, some annuli irregular.
Male: Habitus C-shaped (20\%) to spiral $(80 \%)$. Lip region $6 \pm 0.5(5-6) \mu \mathrm{m}$ high and $8 \pm 0.4(8-9) \mu \mathrm{m}$ wide; with six to seven annuli, basal annuli bulging out. Anterior cephalids three to four annuli posterior to basal ring, posterior cephalids 12 to 14 annuli posterior to anterior cephalids. Stylet more slender than that of female; stylet knobs $2 \pm 0.4(1-2) \mu \mathrm{m}$ high and $4 \pm 0.8$ (4-5) $\mu \mathrm{m}$ wide, anterior faces flattened $(50 \%)$ or indented $(50 \%)$. Position of DGO $4 \pm 0.6(3-4) \mu \mathrm{m}$ behind stylet knobs. Median bulb oval, $15 \pm 2.3(13-19) \mu \mathrm{m}$ long and $10 \mu \mathrm{m}$ wide; valve $3 \pm 0.3$ (3-4) $\mu \mathrm{m}$ long and $3 \pm 0.8$ (2-4) $\mu \mathrm{m}$ wide. Length of oesophagus $146 \pm 9.3(133-153) \mu \mathrm{m}$, with length to end of glands $169 \pm 15.4$ (147184) $\mu \mathrm{m}$. Oesophagus with $29 \pm 8.9$ (1839) $\mu \mathrm{m}$ long ventral or ventro-lateral overlap. Excretory pore $129 \pm 5.4(121-136) \mu \mathrm{m}$ from front, i.e. at $15 \pm 0.7$ (14-16) \% of body length. Hemizonid one to three annuli long, located two to three annuli anterior to excretory pore. Hemizonion half to one annuli long, located 10 to 13 annuli posterior to excretory pore. Fasciculi present. Width of annuli at midbody $2 \mu \mathrm{m}$. Body width at excretory pore $20 \pm 1.8(18-23) \mu \mathrm{m}$, at midbody $24 \pm 3.3$ (21-29) $\mu \mathrm{m}$ and at cloaca 17 $\pm 3.3(15-24) \mu \mathrm{m}$. Lateral field $6 \pm 0.3$ (6-7) $\mu \mathrm{m}$ wide; outer two lines areolated anterior to and opposite bursa, crenate or incompletely areolated on body. Phasmids located posterior to cloaca. Margin of bursa almost straight, flattened. Tail $31 \pm 4.9$ (26-39) $\mu \mathrm{m}$ long, conical with rounded projection.

\section{Diagnosis}

H. curatus sp. $\mathrm{n}$. is characterised by stylet length (42-46 $\mu \mathrm{m}$ in females, $37-40 \mu \mathrm{m}$ in males), presence of two rudimentary subdorsal and two rudimentary subventral lobes on the labial disc, first lip annulus divided into six sectors, presence of fasciculi and presence of males.

\section{Relationships}

A conspicuous character of $H$. curatus sp. n. is the short DGO $(5-9 \mu \mathrm{m})$ in females and $(3-4 \mu \mathrm{m})$ in males. This short DGO fit into 

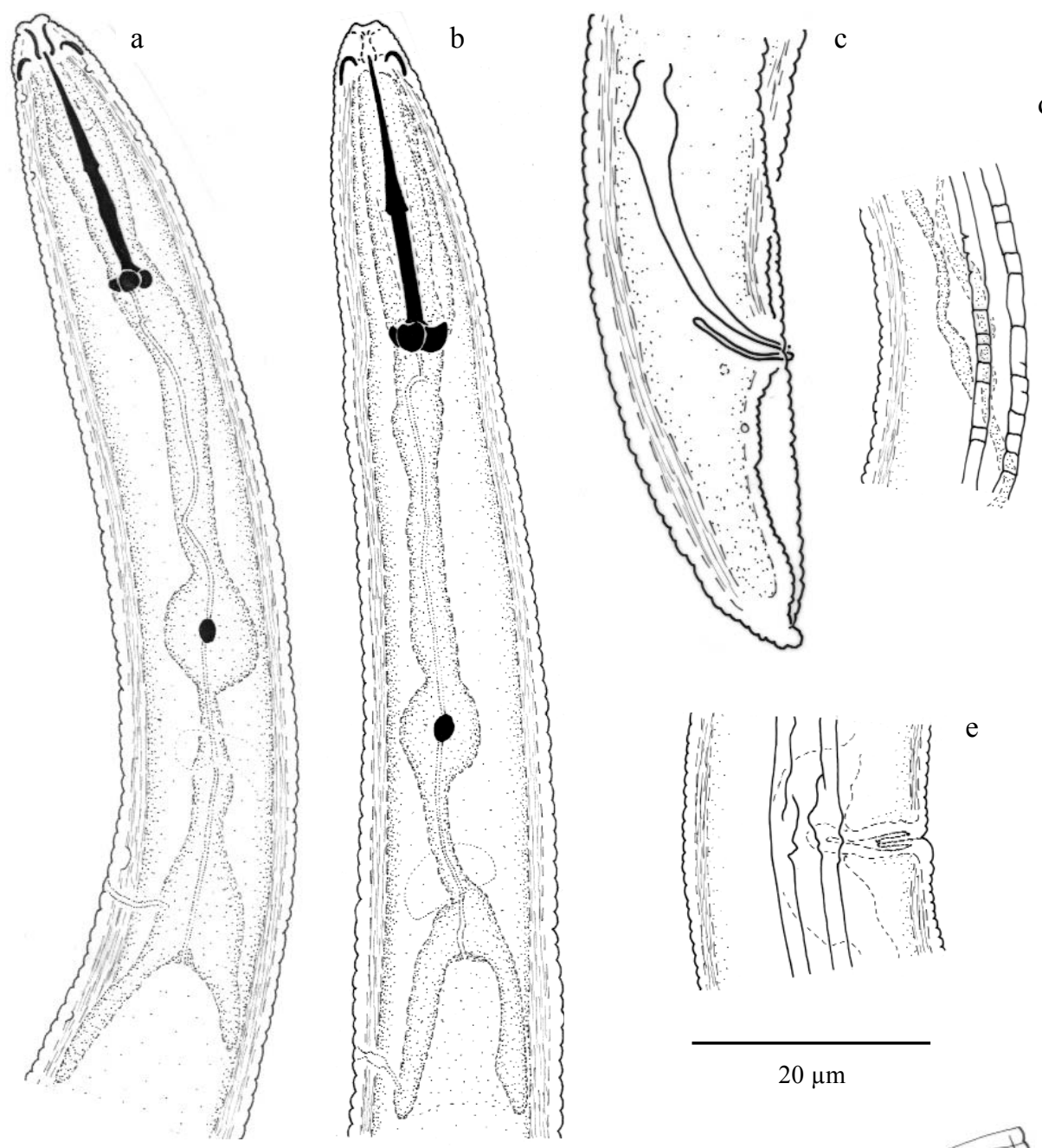

d
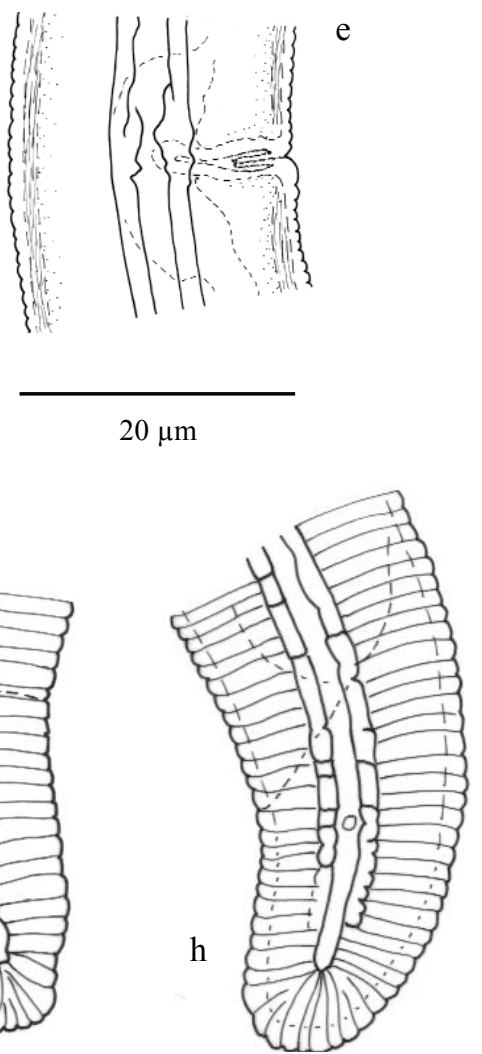

Fig. 2. Helictylenchus curatus sp. n. Female. (a) Oesophageal region. (d) Lateral field and fasciculi. (e) Vulval region. (f-h) Posterior part of body. Male. (b) Oesophageal region. (c) Posterior part of body. 
the diagnosis for the genus where the DGO varies from $3 \mu \mathrm{m}$ (H. labiatus in Yeates \& Wouts 1992) to $23 \mu \mathrm{m}$ (H. erythrinae in Marais 2001). According to Fortuner (1984), this character has little value for identification, therefor the DGO was not used as a differentiating character. The stylet length has the smallest coefficient of variation among the quantitative characters (Fortuner 1979; Fortuner et al. 1981). There is a group of species in Helicotylenchus characterised by long bodies and stylets, the respective measurements ranging from $800 \mu \mathrm{m}$ to $1300 \mu \mathrm{m}$ and from $35 \mu \mathrm{m}$ to $46 \mu \mathrm{m}$. Nine species are included in this group: $H$. arliani Khan, Singh \& Lal, 1998, H. canalis Sher, 1966, H. coomansi Sharafati-Ali \& Loof, 1975, H. dolichodoryphorus Sher, 1966, H. kashmirensis Fotedar \& Handoo, 1974, H. macrostylus Marais \& Quénéhervé, 1996, H. orthosomaticus Siddiqi, 1972, H. paracanalis Sauer \& Winoto, 1975, H. rohtangus Jairajpuri \& Baqri, 1973 and H. tunisiensis Siddiqi, 1963 (Jairajpuri \& Baqri 1973; Fotedar \& Handoo 1974; Kepenekci \& Ökten 1996; Khan et al. 1998; Marais 1998; Marais \& Quénéhervé 1996; Marais et al. 2003; Sharafati-Ali \& Loof 1975; Sher 1966; Siddiqi 1972; Van den Berg \& Kirby 1979). H. curatus sp. n. was compared with three of these species, where the stylet lengths are more than $40 \mu \mathrm{m}$ i.e. H. canalis, $H$. coomansi and $H$. macrostylus. The new species can be separated from $H$. canalis in lip region shape (truncate with basal annuli bulging out vs flattened to rounded, not set off), labial disc characteristics (rectangular in en face view, with two rudimentary subdorsal and two rudimentary subventral lobes vs oval in en face view without any lobes), first lip annulus divided vs not divided, mean body length $(1040 \mu \mathrm{m} v s 859 \mu \mathrm{m})$, position of excretory pore from front $(142-172 \mu \mathrm{m} v s$ 121-143 $\mu \mathrm{m})$, mean tail length $(24 \mu \mathrm{m} v s$ $20 \mu \mathrm{m}$ ), position of phasmids (opposite anus to five annuli posterior to anus vs two to seven annuli anterior to anus) and presence of males vs absence of males. Females of the new species can be distinguished from $H$. coomansi females in lip region shape (truncate with basal annuli bulging out vs continuous), labial disc characteristics (with two rudimentary subdorsal and two rudimentary subventral lobes present vs without any lobes), number of lip annuli (six to seven $v s$ four to five), presence $v s$ absence of fasciculi, a-value (17.5-27.5 vs 35-40), stylet length (42-46 $\mu \mathrm{m}$ vs 39-42 $\mu \mathrm{m})$, m-value (52-56\% vs 48-50\%), position of phasmids (opposite anus to five annuli posterior to anus vs five to eight annuli anterior to anus) and tail form (asymmetrical, more curved dorsally, with rounded end vs straight, tapering dorsally with unstriated ventral portion). The males differ in body length (760$944 \mu \mathrm{m}$ vs 1190-1260 $\mu \mathrm{m}$ ), position of excretory pore from front (121-126 $\mu \mathrm{m} v \mathrm{~s}$ $161 \mu \mathrm{m})$ and tail length $(26-39 \mu \mathrm{m} v s 41 \mu \mathrm{m}$ - calculated from paratype material.). The new species can be separated from $H$. macrostylus in presence of fasciculi vs absence, m-value (52-56 \% vs 42-45\%), lip region shape (truncate with basal annuli bulging out $v s$ continuous), labial disc characteristics (rectangular, with two rudimentary subdorsal and two rudimentary subventral lobes $v s$ round with no lobes), first lip annulus divided vs not divided.

Type locality and habitat

Collected from Cyclopia plicata Kies at Haarlem, Langkloof, Western Cape Province $\left(33^{\circ} 44^{\prime} \mathrm{S}, 2^{\circ} 20^{\prime} \mathrm{E}\right)$ in a Mountain Fynbos vegetation type by $\mathrm{H}$. Hugo.

Type material

Holotype female (slide number 36524), six female paratypes and seven male paratypes (slide 36525-36528) deposited in the National Collection of Nematodes, Biosystematics Division, ARC-Plant Protection Research Institute, Pretoria, South Africa.

Etymology

From the Latin cura, meaning to care, in recognition of Liezel Scheepers for her invaluable assistance. 


\section{Acknowledgements}

We thank N.H. Buckley and E. van Niekerk (ARCPPRI) for technical assistance, the various farmers, foresters and officers in charge for their help in collecting the soil samples and P.A.A. Loof (Landbouwuniversiteit Wageningen) for the loan of H. coomansi paratypes.

\section{References}

DeCraEmer, W. 1995. The family Trichodoridae: Stubby root and virus vector nematodes. Dorderecht: Kluwer.

FORTUNER, R. 1979. Morphometrical variability in Helicotylenchus Steiner, 1945. I. The progeny of a single female. Revue de Nématologie 2: 179-202.

FORTUNER, R. 1984. Morphometrical variability in Helicotylenchus Steiner, 1945. 6: Value of the characters used for specific identification. Revue de Nématologie 7: 245-264.

Fortuner, R., G. Merny \& C. Roux. 1981. Morphometrical variability in Helicotylenchus Steiner, 1945. 3: Observations on African populations of Helicotylenchus dihystera and considerations on related species. Revue de Nématologie 4: 235-260.

Fotedar, D.N. \& Z.A. Handoo. 1974. Two new species of Helicotylenchus (Hoplolaiminae: Nematoda) from Kashmir, India. Journal of Science Kashmir University 2: 57-62.

HeYNS, J. 1971. A guide to the plant and soil nematodes of South Africa. Cape Town: Balkema.

Hooper, D.J. \& K. Evans. 1993. Extraction, identification and control of plant nematodes. Pp. 1-59. In: Evans, K., D.L. Trudgill \& J.M. Webster (eds.). Plant parasitic nematodes in temperate agriculture. Wallingford: CAB International.

HunT, D.J. 1993. Aphelenchida, Longidoridae and Trichodoridae: Their systematics and bionomics. Wallingford: CAB International.

JAIRAJPURI, M.S. \& Q.H. BAQRI. 1973. Nematodes of high altitudes in India. I. Four new species of Tylenchida. Nematologica 19: 19-30.

JENKINS, W.R. 1964. A rapid centrifugal-flotation technique for separating nematodes from soil. Plant Disease Reporter 48: 692.

KepenekcI, I. \& M.E. ÖKten. 1996. Beypazari (Ankara) Ilçesi'nde havuf ile münabeye giren domates ekiliú alanlarinda saptanan Helicotylenchus (Tylenchida, Hoplolaimidae) cinsine bagli türler. Türk Entomoloji Dergisi 20: 137-148.

Khan, E., M. Singh \& M. LaL. 1998. Four new species of tylenchids (Nematoda: Tylenchida) from Nepal. International Journal of Nematology 8: 27-32.

Koen, H. \& J.P. Furstenberg. 1970. 'n Beknopte laboratoriumhandleiding vir nematologie. Pretoria: Departement van Landbou-Tegniese Dienste. (Tegniese Mededeling 92.)

KLeynhans, K.P.N. 1991. The root-knot nematodes of South Africa. Pretoria: Department of Agricultural Development, South Africa. (Technical Communications no. 195.)

KLEYNHANS, K.P.N. 1997. Collecting and preserving nematodes. A manual for a SAFRINET course in practical nematology. ARC-Plant Protection Research Institute. Pretoria.

Kleynhans, K.P.N., E. van den Berg, A. Swart, M. Marais \& N.H. BuCKLeY. 1996. Plant nematodes in South Africa. Pretoria: ARC-Plant Protection Research Institute. (Plant Protection Research Institute Handbook; no. 8.)

Low, A.B. \& A.G. ReBeLO. 1996. Vegetation of South Africa, Lesotho and Swaziland. A companion to the Vegetation Map of South Africa, Lesotho and Swaziland. Pretoria: Department of Environmental Affairs \& Tourism.

Maggenti, A.R., M. Luc, D.J. RAski, R. Fortuner \& E. Geraert. 1988. A reappraisal of Tylenchina (Nemata). 11. List of generic and suprageneric taxa, with their junior synonyms. Revue de Nématologie 11: 177-188.

MARAIS, M. 1993. On some Helicotylenchus Steiner, 1945 from South Africa. Phytophylactica 25: 21-35.

MaraIs, M. 1998. Some species of Helicotylenchus Steiner, 1945 from South Africa (Nematoda: Hoplolaimidae). Fundamental and Applied Nematology 21: 327-352.

Marais, M. 2001. A monograph of the genus Helicotylenchus Steiner, 1945 (Nemata: Hoplolaimidae). Ph.D. Agric. dissertation, University of Stellenbosch, Stellenbosch.

MARAIS, M. \& N.H. BuCKLEY. 1992. External morphology of eight South African Helicotylenchus species (Hoplolaimidae: Nemata). Phytophylactica 24: 297-306.

MARAIS, M. \& P. QuÉNÉHERvÉ. 1996. Helicotylenchus macrostylus n.sp. (Hoplolaimidae: Nemata) from French Guiana with notes on three known species. Nematropica 26: 39-45.

MARAIS, M. \& P. QuÉNÉHERVÉ. 1999. A new species of Helicotylenchus from French Guiana, with notes on two known species (Nemata: Hoplolaimidae). Journal for Nematode Morphology and Systematics 2: 81-88.

Marais, M., P. Quénéhervé, L.R. Tiedt \& A.J. MeYer. 2003. Description of Helicotylenchus marethae n.sp., with redescriptions of some Helicotylenchus species (Nemata: Hoplolaimidae) from South Africa and Martinique. Journal 
of Nematode Morphology and Systematics 5: 1-19. (2002 published in 2003)

Marais, M. \& A. SwarT. 1999. Plant nematodes in the Lottering Plantation, Eastern Cape Province. South African Forestry Journal 186: 77-79.

Marais, M. \& E. VAN DEN BERG, P. QuÉNÉHERVÉ \& L.R. TIEDT. 2000. Description of Helicotylenchus kermarreci n.sp., with notes on some Helicotylenchus Steiner, 1945 and a Rotylenchus Filip'ev, 1936 species (Nemata: Hoplolaimidae) from the Guadeloupe Islands, French West Indies. Journal for Nematode Morphology and Systematics 2: 159-172. (1999 published in 2000).

Netscher, C. \& J.W. Seinhorst. 1969. Propionic acid better than acetic acid for killing nematodes. Nematologica 15: 286.

Rebelo, A.G. 1996a. Renosterveld. Pp. 63. In: Low, A.B. \& A.G. ReBElo. Vegetation of South Africa, Lesotho and Swaziland. A companion to the Vegetation Map of South Africa, Lesotho and Swaziland. Pretoria: Department of Environmental Affairs \& Tourism.

Rebelo, A.G. 1996b. Fynbos. Pp. 68-70. In: Low, A.B. \& A.G. ReBelo. Vegetation of South Africa, Lesotho and Swaziland. A companion to the Vegetation Map of South Africa, Lesotho and Swaziland. Pretoria: Department of Environmental Affairs \& Tourism.

SAuER, M. R. \& R. Winoto. 1975. The genus Helicotylenchus Steiner, 1945 in West Malaysia. Nematologica 21: 341-350.

SEINHORST, J.W. 1959. A rapid method for the transfer of nematodes from fixative to anhydrous glycerin. Nematologica 4: 67-69.

Sharafati-Ali, S. \& P.A.A. Loof. 1975. Two new species of Helicotylenchus Steiner, 1945 (Nema- toda: Hoplolaimidae). Nematologica 21: 207-212.

SHER, S.A. 1966. Revision of the Hoplolaiminae (Nematoda) VI. Helicotylenchus Steiner, 1945. Nematologica 12: 1-56.

SIDDIQI, M.R. 1972. On the genus Helicotylenchus Steiner, 1945 (Nematoda: Tylenchida), with descriptions of nine new species. Nematologica 18: 74-91.

VAN DEN Berg, E. \& M. F. Kirby. 1979. Some spiral nematodes from the Fiji Islands (Hoplolaimidae: Nematoda). Phytophylactica 11: 99-109.

VAn den BerG, E. \& M. Marais. 1995. New species of Tylenchina Chitwood (Nemata) from the Amazonas province, Brazil. African Plant Protection 1: 25-39.

VAn den Berg, E., M. Marais, S. Gaidashova \& L.R. TIEDT. 2003. Hoplolaimidae Filip'ev, 1934 (Nemata) from Rwandan banana fields. African Plant Protection 9: 31-42.

VAn Den Berg, E., M. Marais \& L.R. Tiedt. 2003. Plant nematodes in South Africa. 5. Plant nematodes from the Gouekrans area of the Swartberg nature Reserve, with descriptions of one new and one known Hemicycliophorionae (Nemata). African Plant Protection 9: 43-52.

VAn Den BerG, E. \& L.R. TIEDT. 2000. Hemicycliophora stiaani sp.n. with notes on three tylenchid (Nemata) species from South Africa. Journal of Nematode Morphology and Systematics 2: 137-148. (1999 published 2000).

YeAtes, G.W. \& W.M. Wouts. 1992. Helicotylenchus spp. (Nematoda: Tylenchida) from managed soils in New Zealand. New Zealand Journal of Zoology 19: 13-23. 